\title{
Fatores de risco para estresse e transtornos mentais em farmacêuticos e auxiliares de farmácia
}

\author{
Risk factors for stress and mental disorders in pharmacists and \\ pharmacist aides
}

\author{
Evandro C. S. Vilela ${ }^{1}$, Leonardo R. Soares ${ }^{2}$, Alex S de Gusmão ${ }^{2}$, \\ Rafael A. T. Torres ${ }^{2}$, Eduardo C. Sá ${ }^{2}$
}

\begin{abstract}
Vilela ECS, Soares LR, Gusmão AS, Torres RAT, Sá EC. Fatores de risco para estresse e transtornos mentais em farmacêuticos e auxiliares de farmácia. Saúde, Ética \& Justiça. 2015;20(2):77-83.

RESUMO: Introdução: Os transtornos mentais e o estresse relacionados ao trabalho podem ser provenientes de situações inerentes ao processo de trabalho e sua organização. Dentre os profissionais mais afetados estão aqueles que integram os serviços de saúde, envolvendo farmacêuticos e auxiliares de farmácia. O papel do farmacêutico e, consequentemente, a assistência farmacêutica, sofreram profundas mudanças durante o século XX que culminaram em suas atuais atribuições que, associadas ao fato de estarem na interface entre a prescrição e a dispensação de medicamentos com a responsabilidade de diminuir riscos terapêuticos, envolvidos em questões de cunho legal e clínico/social, submetem diariamente os profissionais a fatores estressores. Objetivo: Identificar os fatores de risco mais prevalentes para estresse e transtornos mentais relacionados ao trabalho em farmacêuticos e auxiliares de farmácia. Metodologia: Realizada uma revisão bibliográfica, utilizando os descritores "Farmacêuticos/ Auxiliares de farmácia" ("Pharmacist / Pharmacist'Aides"), "Transtorno mental" ("Mental Disorders"), "Estresse" ("Stress") e "Esgotamento profissional" ("Burnout") nas bases de dados: PubMed, ScieLo, BVS e Science Direct. Resultados/Discussão: Foram encontrados doze artigos: sete abordavam fatores estressores relativos à profissão; cinco abordavam especificamente transtornos mentais. Dentre os primeiros, seis deles são de literatura internacional, sendo que o único artigo nacional é o de Bastos et al. (2010), que expressa possíveis fatores estressores após análise de uma série de entrevistas com farmacêuticos comunitários do Rio de Janeiro. Os transtornos de origem mental são abordados em estudos com alunos de graduação em farmácia (Hunt et al., 2013) que mostram casos de depressão e transtorno obsessivo-compulsivo relacionados a distúrbios do sono e anedonia. Conclusão: Os fatores mais prevalentes encontrados foram: extensa jornada de trabalho, número insuficiente de profissionais no ambiente laboral e diversas interrupções durante turno de trabalho.
\end{abstract}

DESCRITORES: Farmacêuticos/psicologia; Auxiliares de farmácia/psicologia; Transtornos mentais; Esgotamento profissional; Fatores de risco.

\footnotetext{
${ }^{1}$ Projeto Região Oeste - Fundação Faculdade de Medicina, Hospital das Clínicas da Faculdade de Medicina da Universidade de São Paulo.

${ }^{2}$ Departamento de Medicina Legal, Ética Médica, Medicina Social e do Trabalho da Faculdade de Medicina da Universidade de São Paulo.

Endereço para correspondência: Evandro C S Vilela: E-mail: vilela80@uol.com.br
} 


\section{INTRODUÇÃO}

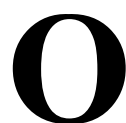

papel do farmacêutico e, consequentemente, a assistência farmacêutica, sofreram profundas mudanças durante o século XX em que podem ser delimitados três períodos, segundo Hepler e Strand ${ }^{1}$ : o tradicional, o de transição e o de desenvolvimento da atenção ao paciente.

No primeiro momento, o tradicional, o farmacêutico era aquele que preparava e vendia os medicamentos, muitas vezes prescrevendo-os e orientando sobre o uso dos mesmos, mantendo contato direto com seus clientes. O vertiginoso desenvolvimento industrial desencadeou mudanças na produção medicamentosa, conferindo-lhe caráter técnico industrial e atrelou à atividade farmacêutica um caráter mercantilista, expresso na Lei 5.991/73. ${ }^{2}$ A transição para esse modelo, no qual o estabelecimento comercial farmacêutico era voltado ao lucro, em cuja política de entregar ao paciente medicamentos desenfreadamente $^{3}$ e propagandas desmedidas eram difundidas ${ }^{4}$, acarretou em perda de autonomia, prestígio e função enquanto agente social do profissional da farmácia, correspondendo à segunda fase. Em meados da década de 60, o incremento da farmacovigilância ${ }^{5} \mathrm{e}$ da preocupação com o uso racional de medicamentos ${ }^{6}$, além dos malefícios à qualidade de vida que os mesmos podem gerar $^{1}$, impulsionaram o surgimento de uma nova prática farmacêutica, amplamente debatida após a "Declaração de Tóquio", de 1993. ${ }^{7}$

$\mathrm{O}$ retorno das funções relativas à atenção ao paciente converge nas atuais funções do farmacêutico, que englobam um caráter técnico-social ${ }^{8}$, garantindo ao mesmo papel de agente comunitário de saúde, e expressam a terceira fase da assistência farmacêutica ${ }^{1}$. Atualmente, são consideradas algumas das boas práticas desempenhadas por um profissional da farmácia: promoção do uso racional de medicamentos, com a avaliação da posologia e da via de administração; esclarecimentos sobre as contra indicações e os efeitos adversos dos mesmos, com o objetivo de que haja melhor adesão ao tratamento por parte dos pacientes ${ }^{9}$, além de avaliar se o medicamento é viável economicamente para aquela população ${ }^{10}$, criticando as propagandas desmedidas de novas fórmulas e a pressão exercida por certos laboratórios para a prescrição das mesmas. ${ }^{11}$

Por estarem na interface entre a prescrição e a dispensação de medicamentos, com responsabilidade de diminuir riscos terapêuticos e, consequentemente, envolvidos em questões de cunho legal e clínico/ social ${ }^{12}$, imersos no mercantilismo desenvolvido pela farmácia ${ }^{13} \mathrm{e}$, em países como Estados Unidos e Inglaterra, agregando funções relativas à prescrição medicamentosa suplementar ${ }^{14}$ e ajuste terapêutico ${ }^{15}$, os farmacêuticos e os auxiliares de farmácia estão submetidos diariamente a fatores estressores.

$\mathrm{O}$ estresse, agregado à função e às condições internas e externas ao ambiente laboral, pode culminar no desenvolvimento de transtornos mentais, já descritos durante a formação acadêmica dos profissionais da farmácia ${ }^{16}$, e devem ser abordados minuciosamente devido à sua importância para a saúde do trabalhador.

Tanto os transtornos mentais como o estresse relacionados ao trabalho podem ser provenientes de situações inerentes ao processo de trabalho e sua organização, envolvendo divisão e parcelamento das tarefas, as políticas de gerenciamento das pessoas, assédio moral e a estrutura hierárquica organizacional ${ }^{17}$.

No Brasil, em 2013, segundo o anuário estatístico do Ministério da Previdência Social ${ }^{18}$, esses transtornos corresponderam à terceira principal causa de concessão de benefício auxílio-doença, superados apenas por distúrbios osteomusculares e lesões envolvendo causas externas, representando 209 mil novos benefícios, 10\% do total. Dentre os profissionais mais afetados por transtornos mentais estão os que integram os serviços de saúde ${ }^{19}$, envolvendo inclusive os farmacêuticos e os auxiliares de farmácia, foco deste estudo.

\section{OBJETIVO}

Identificar os fatores de risco mais prevalentes para estresse e transtornos mentais relacionados ao trabalho em farmacêuticos e auxiliares de farmácia.

\section{METODOLOGIA}

Foi realizada uma revisão bibliográfica, nos meses de março a junho de 2015, utilizando os descritores "Farmacêuticos" ("Pharmacist"), Auxiliares de farmácia" ("Pharmacist's Aides"), "Transtorno mental" ("Mental Disorders"), "Estresse" ("Stress") e "Esgotamento profissional" ("Burnout") nas bases de dados: PubMed, SciELO, BVS e Science Direct. Foram excluídos os artigos que não estavam em língua inglesa ou portuguesa, os repetidos em mais de uma base, aqueles não disponíveis de forma gratuita nas bases de dados da Universidade de São Paulo e os que não versavam sobre o tema de estudo.

\section{RESULTADOS}

Foram selecionados doze artigos (Tabela 1). Os transtornos e seus respectivos fatores de risco, além de locais de atuação dos profissionais de farmácia, estão descritos na Tabela 2. 
Vilela ECS et al. Fatores de risco para estresse e transtornos mentais em farmacêuticos e auxiliares de farmácia.

Tabela 1. Relação de artigos encontrados segundo os seus autores, ano de publicação, local de origem e título

\begin{tabular}{|c|c|c|c|}
\hline Autor & Ano & Local do estudo & Título do artigo \\
\hline Chui et al. ${ }^{20}$ & 2014 & Estados Unidos & $\begin{array}{l}\text { The association of subjective workload dimensions on quality of care and pharmacist quality of } \\
\text { work life }\end{array}$ \\
\hline $\begin{array}{l}\text { Johnson et } \\
\text { al. }^{21}\end{array}$ & 2014 & Reino Unido & The relationships among work stress, strain and self-reported errors in UK community pharmacy \\
\hline Lea et al. ${ }^{22}$ & 2012 & Reino Unido & $\begin{array}{l}\text { Workload and its impact on community pharmacists' job satisfaction and stress: a review of the } \\
\text { literature }\end{array}$ \\
\hline Calgan et al..$^{23}$ & 2011 & Turquia & Community pharmacists' burnout levels and related factors: an example from Turkey \\
\hline Hassell et al. ${ }^{24}$ & 2011 & Reino Unido & $\begin{array}{l}\text { Workload in community pharmacies in the UK and its impact on patient safety and pharmacists, } \\
\text { well-being: a review of the evidence }\end{array}$ \\
\hline $\begin{array}{l}\text { Rothmann et } \\
\text { al. }{ }^{25}\end{array}$ & 2011 & África do Sul & Work-related well-being of South African hospital pharmacists \\
\hline Bastos et al. ${ }^{13}$ & 2010 & Brasil & $\begin{array}{l}\text { As percepções dos farmacêuticos sobre seu trabalho nas farmácias comunitárias em uma região do } \\
\text { estado do Rio de Janeiro }\end{array}$ \\
\hline Holden et al. ${ }^{26}$ & 2010 & Estados Unidos & $\begin{array}{l}\text { Effects of mental demands during dispensing on perceived medication safety and employee well- } \\
\text { being: A study of workload in pediatric hospital pharmacies }\end{array}$ \\
\hline $\begin{array}{l}\text { Gaither et } \\
\text { al. }^{27}\end{array}$ & 2008 & Estados Unidos & $\begin{array}{l}\text { A modified model of pharmacists' job stress: The role of organizational, extra-role, and individual } \\
\text { factors on work-related outcomes }\end{array}$ \\
\hline $\begin{array}{l}\text { Gidman et } \\
\text { al. }^{28}\end{array}$ & 2007 & Reino Unido & $\begin{array}{l}\text { The impact of increasing workloads and role expansion on female community pharmacists in the } \\
\text { United Kingdom }\end{array}$ \\
\hline Lapane et al. ${ }^{29}$ & 2004 & Estados Unidos & $\begin{array}{l}\text { Baseline job satisfaction and stress among pharmacists and pharmacy technicians participating in } \\
\text { the Fleetwood Phase III Study }\end{array}$ \\
\hline $\begin{array}{l}\text { Gupchup et } \\
\text { al. }^{30}\end{array}$ & 1998 & $\begin{array}{l}\text { Múltiplas } \\
\text { localizações }\end{array}$ & Burnout in a sample of HMO pharmacists using the Maslach Burnout Inventory \\
\hline
\end{tabular}

Tabela 2. Relação dos artigos encontrados que citam os fatores de risco para transtornos mentais e o local de atuação profissional dos profissionais de farmácia

\begin{tabular}{|lllll}
\hline Autor & Ano & $\begin{array}{l}\text { Local de atuação do } \\
\text { farmacêutico }\end{array}$ & $\begin{array}{l}\text { Transtorno } \\
\text { abordado }\end{array}$ & Fatores de risco para transtornos abordados \\
\hline $\begin{array}{l}\text { Chui } \\
\text { al. }{ }^{20}\end{array}$ & et 2014 & Farmácias comunitárias & Burnout & $\begin{array}{l}\text { Extenuantes jornadas de trabalho; Número insuficiente de funcionários; } \\
\text { Altas demandas profissionais; Alta demanda de concentração e esforço } \\
\text { mental; Satisfação no trabalho. }\end{array}$
\end{tabular}

\begin{tabular}{|c|c|c|c|c|c|}
\hline $\begin{array}{l}\text { Johnson et } \\
\text { al. } .^{21}\end{array}$ & 2014 & Farmácias comunitárias & Stress & & $\begin{array}{l}\text { Sobrecarga de trabalho; Extenuantes jornadas de trabalho; Falta de } \\
\text { estabilidade; Controle sobre o trabalho realizado; Equilíbrio entre vida } \\
\text { pessoal e profissional; Relação interpessoal; Erros de prescrição; Inadequação } \\
\text { de equipamentos e recursos. }\end{array}$ \\
\hline Lea et al..$^{22}$ & 2012 & Farmácias comunitárias & Stress & & $\begin{array}{l}\text { Extenuantes jornadas de trabalho, interrupções frequentes, isolamento } \\
\text { profissional; Número insuficiente de funcionários. }\end{array}$ \\
\hline $\begin{array}{l}\text { Calgan et } \\
\text { al. }^{23}\end{array}$ & 2011 & Farmácias comunitárias & Burnout & & $\begin{array}{l}\text { Idade; Estado civil; Satisfação profissional; Jornadas de trabalho excessivas; } \\
\text { Alta demanda de trabalho com pouco tempo para sua realização; Stress; } \\
\text { Experiência profissional. }\end{array}$ \\
\hline $\begin{array}{l}\text { Hassel et } \\
\text { al. } .^{24}\end{array}$ & 2011 & Farmácias comunitárias & Stress & & $\begin{array}{l}\text { Extenuantes jornadas de trabalho e trabalho excessivo; Metas de trabalho } \\
\text { inatingíveis; Acatar funções não correspondentes à de um farmacêutico; } \\
\text { Interrupções contínuas; Número insuficiente de funcionários. }\end{array}$ \\
\hline $\begin{array}{l}\text { Rothmann } \\
\text { et al. } .^{25}\end{array}$ & 2011 & Farmácias hospitalares & $\begin{array}{l}\text { Burnout } \\
\text { Stress }\end{array}$ & & $\begin{array}{l}\text { Medicamentos não disponíveis através de fornecedores, interrupções } \\
\text { frequentes, companheiros de trabalho não trabalhando, salário e funcionários } \\
\text { insuficientes como fontes de stress; Stress e ausência de estratégias para } \\
\text { combate-lo, ausência de recursos, altas demandas profissionais, baixa } \\
\text { religiosidade }\end{array}$ \\
\hline
\end{tabular}

\section{Continua}




\section{Continuação}

\begin{tabular}{|c|c|c|c|c|}
\hline Autor & Ano & $\begin{array}{l}\text { Local de atuação do } \\
\text { farmacêutico }\end{array}$ & $\begin{array}{l}\text { Transtorno } \\
\text { abordado }\end{array}$ & Fatores de risco para transtornos abordados \\
\hline $\begin{array}{l}\text { Bastos et } \\
\text { al. }^{13}\end{array}$ & 2010 & Farmácias comunitárias & Stress & $\begin{array}{l}\text { Mudanças contínuas na legislação para dispensação de medicamentos; } \\
\text { Dificuldades de comunicação com outros profissionais; Dificuldades } \\
\text { com legibilidade de prescrições; Conflitos entre interesse econômico da } \\
\text { farmácia e melhor indicação medicamentosa; Desvalorização salarial; Não } \\
\text { reconhecimento pela equipe de saúde; Conflitos com supervisores; Número } \\
\text { insuficiente de trabalhadores; Impossibilidade de continuar estudando; Falta } \\
\text { de assistência aos profissionais pelos órgãos governamentais e sindicatos; } \\
\text { Inadequação do ambiente de trabalho. }\end{array}$ \\
\hline
\end{tabular}

\begin{tabular}{lllll}
\hline $\begin{array}{l}\text { Holden et } \\
\text { al. }{ }^{26}\end{array}$ & Farmácias hospitalares & Burnout & $\begin{array}{l}\text { Interrupções contínuas em ambiente de trabalho; Necessidade de dividir } \\
\text { a atenção entre vários assuntos simultaneamente; Pressa na realização de } \\
\text { tarefas. }\end{array}$ \\
\hline $\begin{array}{l}\text { Gaither et } \\
\text { al. }{ }^{27}\end{array}$ & & Sem especificação & Stress & $\begin{array}{l}\text { Dificuldades em relações interpessoais; Falta de feedback e incentivo de } \\
\text { supervisores; }\end{array}$
\end{tabular}

\begin{tabular}{lllll}
\hline $\begin{array}{l}\text { Gidman et } \\
\text { al. }{ }^{28}\end{array}$ & 2007 & Farmácias comunitárias & Stress & Extensas jornadas de trabalho. \\
\hline $\begin{array}{l}\text { Lapane et } 2004 \\
\text { al. }^{\mathbf{2 9}}\end{array}$ & & $\begin{array}{l}\text { Farmácias de cuidados } \\
\text { contínuos }\end{array}$ & Stress & $\begin{array}{l}\text { Responsabilidade com o paciente e seu diagnóstico; Receio de errar no } \\
\text { tratamento; Conflitos familiares, com outros profissionais de saúde e } \\
\end{array}$ \\
& & & $\begin{array}{l}\text { supervisores; Interrupções do turno de trabalho por telefone ou pessoas; } \\
\text { Número insuficiente de profissionais trabalhando; Excesso de trabalho; } \\
\text { Salários e oportunidades de crescimento insuficientes; Extensas jornadas de } \\
\text { trabalho. }\end{array}$
\end{tabular}

\begin{tabular}{|c|c|c|c|c|}
\hline $\begin{array}{l}\text { Gupchup } \\
\text { et al. }\end{array}$ & 1998 & Sem especificação & Burnout & $\begin{array}{l}\text { Estado civil; Baixa renda; Patamar hierárquico e anos na prática farmacêutica; } \\
\text { Número de filhos; Número de horas semanais trabalhadas. }\end{array}$ \\
\hline
\end{tabular}

Gidman et al. ${ }^{28}$, em estudo realizado na Inglaterra, abordam os farmacêuticos que trabalham em ambientes comunitários, e identificam os fatores estressores em ambiente de trabalho, tais como: jornadas de trabalho mais longas, incapacidade de exercer sua função de maneira correta, a falta de feedback e de incentivo por parte dos supervisores, fatores abordados também no artigo de Gaither et al. ${ }^{27}$, publicado nos EUA.

Lapane et al. ${ }^{29}$, em seu trabalho realizado na Carolina do Norte, segmentam em três categorias os trabalhadores da farmácia que atendem aos pacientes sob cuidado contínuo: os que trabalham dispensando medicamentos, os auxiliares de farmácia e os supervisores farmacêuticos. Para cada grupo, identificaram diferentes fatores estressores a partir da aplicação de questionários. Ao analisar os 96 participantes do estudo, observaram fontes comuns de estresse para farmacêuticos que trabalham dispensando medicamentos e para os supervisores, como a responsabilidade atrelada ao prognóstico do paciente ou o receio de que um erro poderia ser cometido no tratamento do mesmo. Fator mais comum aos profissionais da dispensação e técnicos seria aquele relacionado ao pequeno número de profissionais no local de trabalho para exercer essa atividade. Nesse estudo foi citado como exemplo de fator comum de estresse aos três grupos de farmacêuticos a grande quantidade de trabalho.

Johnson et al. ${ }^{21}$, em seu estudo com farmacêuticos do Reino Unido, concluíram que estes apresentam sobrecarga no trabalho, ausência de estabilidade ocupacional, inadequação de equipamentos e recursos e falta de controle sobre o trabalho como fatores estressores relacionados ao ambiente da farmácia, confirmando achados semelhantes dos artigos de revisão feitos por Lea et al. ${ }^{22}$ e Hassell et al. ${ }^{24}$ no Reino Unido. Este último revela, ainda, outras fontes de estresse, tais como: a necessidade de atingir metas e o desempenho de funções que distanciam o profissional do contato direto com o paciente.

Em uma série de entrevistas com farmacêuticos de estabelecimentos comunitários do Estado do Rio de Janeiro, Bastos et al. ${ }^{13}$ citam os fatores que geram dificuldades e insatisfação com o trabalho, como mudanças constantes na legislação de dispensação de medicamentos, legibilidade comprometida de receituários médicos, conflitos envolvendo a escolha do 
melhor medicamento para o paciente em contraposição ao mercantilismo exacerbado exercido em farmácias, desvalorização salarial, não reconhecimento pela equipe de saúde e pela população em geral, além de fatores já citados anteriormente, como baixo número de trabalhadores no estabelecimento e conflitos com supervisores.

A síndrome de Burnout é descrita em diversos artigos e possui fatores causais variáveis. Calgan et al. ${ }^{23}$, ao avaliar farmacêuticos comunitários da Turquia, identificaram que essa síndrome está relacionada a fatores estressores no trabalho e a fatores envolvidos na jornada, na experiência profissional e relacionados à idade. $\mathrm{Na}$ avaliação de farmacêuticos americanos, Gupchup et al. ${ }^{30}$ identificaram os parâmetros de despersonalização, exaustão emocional e perda da capacidade laboral, além dos fatores de risco já citados por Calgan et al. ${ }^{23}$. Outros fatores de risco identificados pelos autores foram: o número de filhos e o estado civil dos profissionais, além de número de horas trabalhadas por semana. No estudo em farmacêuticos hospitalares Sul-africanos de Rothmann et al. ${ }^{25}$, os fatores relacionados à síndrome de Burnout eram provenientes dos estressores do ambiente de trabalho (pressão e déficit de recursos), além de fatores pessoais como a incapacidade para evitar estresse e baixa religiosidade.

Holden et al. ${ }^{26}$, ao abordar farmacêuticos de dois hospitais pediátricos americanos, identificaram uma relação direta entre Burnout e interrupções contínuas em ambiente de trabalho, necessidade de dividir a atenção entre vários assuntos simultaneamente e a pressa na realização de tarefas. Já o estudo realizado por Chui et al. ${ }^{20}$, em Wisconsin, associa a síndrome em questão à insatisfação no trabalho em farmácias comunitárias.

\section{DISCUSSÃO}

A avaliação de fatores de risco e patologias associadas a transtornos mentais em farmacêuticos e auxiliares de farmácia se mostra restrita na literatura. Dentre os doze artigos encontrados utilizando os descritores e as bases de dados, sete abordavam fatores estressores relativos à profissão ${ }^{13,21,22,24,27-29}$ e cinco abordavam especificamente transtornos de ordem mental $20,23,25,26,30$.

Dentre os sete artigos que abordam fatores de estresse relacionados aos profissionais de farmácias, $\mathrm{o}$ de Lea et $\mathrm{al}^{22}$ e o de Hassell et al. ${ }^{24}$ são revisões bibliográficas que compartilham diversos artigos em suas referências, apresentando, consequentemente, fatores estressores similares, causando a falsa impressão de estressores semelhantes em trabalhos distintos. Dentre os sete artigos encontrados, seis representam literatura internacional ${ }^{21,22,24,27-29}$, sendo que o único artigo nacional correlato ao objetivo proposto é o de Bastos et al. ${ }^{13}$, que expressa possíveis fatores estressores após uma série de entrevistas com farmacêuticos comunitários do Rio de Janeiro.

Com relação aos artigos que avaliam transtornos mentais específicos, todos os artigos encontrados versam sobre a síndrome de Burnout ${ }^{20,23,25,26,30}$, não havendo literatura abordando demais transtornos mentais específicos aos farmacêuticos e auxiliares de farmácia. Tais transtornos são abordados em alunos de graduação em farmácia, como citado por Hunt et al. ${ }^{16}$, que mostram casos de depressão e transtorno obsessivo-compulsivo relacionados a distúrbios do sono e anedonia.

Apesar de os artigos abordarem farmacêuticos de vários países e, por consequência, com formação e incumbências heterogêneas ${ }^{14,15}$, que estão inseridos em locais distintos, como hospitais ou farmácias comunitárias, e abordando pessoas diferentes em relação à cultura e classe econômica, observa-se como meta comum no trabalho de farmacêuticos: a promoção do uso racional dos medicamentos; a avaliação e o esclarecimento da forma de uso, visando a adesão ao tratamento"; a melhor viabilidade econômica ${ }^{10}$, não se submetendo a pressões da indústria e do comércio. Assim como os objetivos, muitos dos fatores estressores são semelhantes.

O fator de estresse mais prevalente é relacionado à extensa jornada de trabalho ${ }^{20-24,28,29}$, seguido de número insuficiente de profissionais no ambiente da farmácia ${ }^{20,22,24,25,29} \mathrm{e}$ diversas interrupções durante turno laboral ${ }^{22,24-26,29}$. Tais fatores, intrínsecos ao ambiente de trabalho, são consequência do maior contato do farmacêutico com o público, correspondendo à terceira e atual fase da assistência farmacêutica segundo Hepler e Strand $^{1}$, mas atrelam fatores organizacionais relacionados a metas de produção ${ }^{24}$ e lucro dos estabelecimentos farmacêuticos, remetendo ao mercantilismo desenvolvido pela farmácia ${ }^{1}$, levando o profissional a trabalhar cada vez mais e de forma mais intensa, podendo gerar insatisfação profissional $^{20}$, outras fontes de estresse e transtornos mentais.

Outro fator importante é a dificuldade de comunicação com outros profissionais da área de saúde e o sentimento de estar exercendo uma função de importância secundária para o tratamento do paciente. Entraves entre a prescrição e dispensação do medicamento, como a ilegibilidade caligráfica das prescrições ${ }^{13}$, podem acarretar em uma terapêutica errônea e, por conseguinte, levar o profissional a sentir-se responsável por um desfavorável prognóstico do paciente ${ }^{29}$.

Associados ao estresse inerente ao trabalho, devemos considerar também os fatores estressores externos ao ambiente laboral. Religiosidade ${ }^{25}$, conflitos familiares $^{29}$ e estado civil ${ }^{30}$ são exemplos de fontes eventualmente estressoras que devem ser abordadas de forma minuciosa pois, somadas às fontes relativas ao trabalho, convergem na gênese de transtornos mentais. 
Ou seja, a identificação de fatores de estresse não deve se restringir apenas à farmácia, mas deve fazer referência ao farmacêutico, abordando o mesmo de forma integral e com objetivo de prevenção de doenças do aparelho mental.

\section{CONCLUSÃO}

Os fatores de riscos para estresse e transtorno mental mais prevalentes encontrados em farmacêuticos e auxiliares de farmácia foram: extensa jornada de trabalho, número insuficiente de profissionais no ambiente laboral e diversas interrupções durante turno de trabalho. Porém, é de grande importância identificar e avaliar também os demais fatores estressores visando a manutenção da saúde mental e a prevenção de transtornos específicos, como a síndrome de Burnout.

Vilela ECS, Soares LR, Gusmão AS, Torres RAT, Sá EC. Risk factors for stress and mental disorders in pharmacists and pharmacist aides. Saúde, Ética \& Justiça. 2015;20(2):77-83.

\begin{abstract}
Introduction: Mental disorders and work-related stress may result from situations related to the work process and its poor organization. Among the most affected by mental disorders are the professionals that integrate health services, including pharmacists and pharmacy assistants. The role of the pharmacist and the pharmaceutical care suffered major changes during the twentieth century and culminate in their current powers that, associated with the fact of being in the interface between the prescription and dispensing of medications, with a responsibility to reduce treatment risks and consequently involved in legal and medical/social issues, daily submit the professionals to stressor factors. Objective: To identify the most prevalent risk factors for stress and mental disorders related to work of pharmacist and pharmacist' aides. Methodology: a literature review, using the keywords "Pharmacy / Pharmacist "Aides", "Mental Disorders", "Stress" and "Burnout" in the databases: PubMed, SciELO, BVS and Science Direct. Results / Discussion: There were twelve articles, with seven of them stress factors related to the profession and five specifically mental disorders. Among the first, six of them are international literature, and the only national paper is from Bastos et al. (2010), that expresses possible stressors using a series of interviews with community pharmacists in Rio de Janeiro. Mental disorders are covered only undergraduate students in pharmacy, as quoted by Hunt et al. (2013), which show cases of depression and obsessive-compulsive disorder related and sleep disorders and anhedonia. Conclusion: The most prevalent stressor factors found were: long work hours, frequent interruptions and insufficient number of workers.
\end{abstract}

KEY WORDS: Pharmacists; Pharmacist's aides/psychology; Mental disorders; Burnout, professional; Ris factors.

\title{
REFERÊNCIAS
}

1. Hepler CD, Strand LM. Oportunidades y responsabilidades en la Atención Farmacéutica. Pharm Care Esp. 1999;1:3547.

2. Brasil. Presidência da República, Casa Civil, Subchefia para Assuntos Jurídicos. Lei Federal n ${ }^{\circ}$ 5.991, de 17 de dezembro de 1973. Dispõe sobre o Controle Sanitário do Comércio de Drogas, Medicamentos, Insumos Farmacêuticos e Correlatos, e dá outras Providências. Brasília, DF; 1973. Disponível em: http://www.planalto.gov.br/ccivil_03/leis/L5991.htm

3. Barros JAC. Propaganda de medicamentos: atentado à saúde? São Paulo: Hucitec; 1995.

4. Lexchin J. Uma fraude planejada: a publicidade farmacêutica no terceiro mundo. In: Bonfim JRA, Mercucci VL. A construção da política de medicamentos. São Paulo: Hucitec; 1997. p.269-89.

5. Figueras A, Napchan BM, Mendes GB. Farmacovigilância: ação na reação. São Paulo: Centro de Vigilância Sanitária; 2002.

6. Organização Mundial de Saúde (OMS). Promoción del uso racional de medicamentos: componentes centrales. Genebra;2002.
7. Organização Mundial de Saúde (OMS). El papel del farmaceutico en el sistema de atención de salud. Tókio;1993.

8. Bond C. The need for pharmacy practice research. Int $\mathbf{J}$ Pharm Pract. 2006;14:1-2. DOI: http://dx.doi.org/10.1211/ ijpp.14.1.0001.

9. SOBRAVIME - Sociedade Brasileira de Vigilância de Medicamentos. O que é uso racional de medicamentos. São Paulo: Sobravime; 2001.

10. Lloret Callejo A. La figura del farmacéutico de atención primaria: nuevos retos. Rev Clin Med Familia. 2009;2(6):2567.

11. Rozenfeld S. Farmacêutico: profissional de saúde e cidadão. Ciênc Saúde Coletiva. 2008;13(supl):561-8. DOI: http://dx.doi.org/10.1590/S1413-81232008000700002

12. Pepe VLE, Castro CGSO. A interação entre prescritores, dispensadores e pacientes: informação compartilhada como possível benefício terapêutico. Cad Saúde Pública. 2000;16(3):815-22. DOI: http://dx.doi.org/10.1590/S0102$-311 \mathrm{X} 2000000300029$

13. Bastos CRG, Caetano R. As percepções dos farmacêuticos 
sobre seu trabalho nas farmácias comunitárias em uma região do estado do Rio de Janeiro. Ciênc Saúde Coletiva. 2010;15(supl3):3541-50. DOI: http://dx.doi.org/10.1590/ S1413-81232010000900029.

14. George J, McCaig DJ, Bond CM, Cunningham IT, Diack HL, Watson AM, Stewart DC. Supplementary prescribing: early experiences of pharmacists in Great Britain. Ann Pharmacother. 2006;40(10):1843-50.

15. Karpa KD. Pharmacist critique was ill-informed. Ann Pharmacother. 2006;40(7-8):1441-4.

16. Hunt K, Gable KN. Prevalence of depressive symptoms and obsessive-compulsive personality traits among pharmacy students. Curr Pharm Teach Learn.2013; 5(6):541-5.

17. SINAN - Sistema Nacional de Agravos de Notificação. Brasília, DF: Ministério da Saúde; 2010. Disponível em: http://portalsinan.saude.gov.br.

18. Brasil. Ministério da Previdência Social, DATAPREV - Empresa de Tecnologia e Informações da Previdência Social, INSS - Instituto Nacional do Seguro Social. Anuário Estatístico da Previdência Social 2013. Brasília, DF: Secretaria de Políticas de Previdência Social. Departamento do Regime Geral de Previdência Social, Coordenação-Geral de Estatística, Demografia e Atuária; 2013. Disponível em: http://www.mtps.gov.br/dadosabertos/dados-da-previdencia/previdencia-social-e-inss/ anuario-estatistico-da-previdencia-social-aeps.

19. Schaufeli W, Enzmann D. The Burnout companion to study and practice: a critical analysis. Boca Raton (Estados Unidos): CRC Press; 1998.

20. Chui MA, Look KA, Mott DA. The association of subjective workload dimensions on quality of care and pharmacist quality of work life. Res Social Adm Pharm. 2014;10(2):328-40. DOI: http://dx.doi.org/10.1016/j. sapharm.2013.05.007.

21. Johnson SJ, O'Connor EM, Jacobs S, Hassel K, Ashcroft DM. The relationships among work stress, strain and selfreported errors in UK community pharmacy. Res Social Adm Pharm. 2014;10(6):885-95. DOI: http://dx.doi. org/10.1016/j.sapharm.2013.12.003.

22. Lea VM, Corlett SA, Rodgers RM. Workload and its impact on community pharmacists' job satisfaction and stress: a review of the literature. Int J Pharm Pract. 2012; 20(4):259-71. DOI: http://dx.doi.org/10.1111/j.2042-7174.2012.00192.x.

23. Calgan Z, Aslan D, Yegenoglu S. Community pharmacists' burnout levels and related factors: an example from Turkey. Int J Clin Pharm. 2011;33(1):92-100. DOI: http://dx.doi. org/10.1007/s11096-010-9461-2.

24. Hassell K, Seston EM, Schafheutle EI, Wagner A, Eden M. Workload in community pharmacies in the UK and its impact on patient safety and pharmacists' well-being: a review of the evidence. Health Soc Care Commun. 2011;19(6):561-75. DOI: http://dx.doi.org/10.1111/j.1365-2524.2011.00997.x.

25. Rothmann S, Malan M. Work-related well-being of South African hospital pharmacists. SA J Ind Psychol. 2011; 7(1):111. DOI: http://dx.doi.org/10.4102/sajip.v37i1.895.

26. Holden RJ, Patel NR, Scanlon MC, Shalaby TM, Arnold JM, Karsh BT. Effects of mental demands during dispensing on perceived medication safety and employee well-being: a study of workload in pediatric hospital pharmacies. Res Social Adm Pharm. 2010;6(4):293-306. DOI: http://dx.doi. org/10.1016/j.sapharm.2009.10.001.

27. Gaither CA, Kahaleh AA, Doucette WR, Mott DA, Pederson CA, Schommer JC. A modified model of pharmacists' job stress: the role of organizational, extra-role, and individual factors on workrelated outcomes. Res Social Adm Pharm. 2008;4(3):231-43. DOI: http://dx.doi.org/10.1016/j. sapharm.2008.04.001.

28. Gidman WK, Hassell K, Day J, Payne K. The impact of increasing workloads and role expansion on female community pharmacists in the United Kingdom. Res Social Adm Pharm. 2007;3(3):285-302.

29. Lapane K, Hughes C. Baseline job satisfaction and stress among pharmacists and pharmacy technicians participating in the Fleetwood Phase III Study. Consult Pharm. 2004; 19(11):1029-37.

30 - Gupchup GV, Singhal PK, Dole EJ, Lively BT. Burnout in a sample of HMO pharmacists using the Maslach Burnout Inventory. J Manag Care Pharm. 1998;4(5):495-503. DOI: http://dx.doi.org/10.18553/jmcp.1998.4.5.495. 